\title{
Transitioning to Hybrid Radio/Optical Networks: Development of a Flexible Visible Light Communication Testbed
}

\author{
Alexander McBride ${ }^{* \dagger}$, Burim Derveni ${ }^{* \uparrow}$, Jeffrey Proko ${ }^{* \dagger}$, Pekka Kamsula*, Helal Chowdhury*, Tuomo Hanninen*, \\ Juha-Pekka Mäkelä*, Marcos Katz* \\ * Centre for Wireless Communications, University of Oulu, Oulu, Finland \\ $\dagger$ College of Engineering, Drexel University, Philadelphia, Pennsylvania \\ $\$$ Represents equal contribution \\ mkatz@ee.oulu.fi
}

\begin{abstract}
As the number of wireless connected devices grow, the wireless industry is challenged to meet demand while continuing to improve data rates and quality of service $(\mathrm{QoS})$. Visible light communication (VLC) has significant potential to be used as a new high speed layer in future heterogeneous RF networks. This work reviews the major challenges and important applications of hybrid VLC/RF networks, and details a versatile software defined VLCdownlink/RF-uplink experimental testbed. Our hope in this report is to support and aid RF groups as they transition their research interests to include RF/VLC hybrid networks. Given the low cost, minimal hardware requirements, and experimental flexibility, this testbed represents an ideal system to begin RF/VLC hybrid experiments upon. In this context, we offer this report as a unique blend of review, initial experimental testbed observations, and visionary moving forward towards $5 \mathrm{G}$ communication.
\end{abstract}

Keywords-Visible, light, communication, software, defined, radio, frequency, hybrid, review

\section{INTRODUCTION}

Over the last few years, we have witnessed a remarkable increase in the use of smart phones, tablets and other sophisticated wireless devices. The wireless world research forum predicts that 7 trillion wireless devices will serve 7 billion people by 2017 . If this prediction turns into reality, the number of network-connected wireless devices will reach 1000 times the world's population by 2017 [1]. The paradigm of communications will also change. Not only wireless communication between human-to-human, but also common objects may be connected to the Internet. Communication forms will expand from human to human to device-device (also called machine-machine, M2M). As more devices become connected adding to the vast Internet of Things (IoT), the wireless communications industry is challenged to keep up with everincreasing demand. This substantial growth in tele-traffic will entail tremendous increase in spectrum usage [2]. However, the existing best propagation carrier-frequency of $1-2 \mathrm{GHz}$ is congested. Accordingly, this spectrum scarcity challenge has to be tackled by appropriate countermeasures in future research. As data traffic increases in volume, the carbon footprint of using a mobile phone is also increased [3-5]. Moreover, it is commonly agreed that future high data rate wireless services, such as $5 \mathrm{G}$, will be based on exploiting multiple wireless access technologies [6]. Therefore, 5G enabled terminals are also envisioned as multi-standard supporting wireless devices. These multi-standard wireless devices will be equipped with more complex, power-hungry signal processing hardware, imposing a significant threat of using mobile devices for continuous service due to high power consumption by transmission, reception and other processing hardware [7]. In this case, mobile users will be perpetually searching for power outlets rather than network access, which will bind the mobile user to a single location $[2,8]$.

In order to tackle the challenges mentioned above, such as spectrum scarcity, green wireless communication systems and energy efficiency of mobile device, the world mobile and wireless infrastructure community has taken initiatives to meet the demand and has begun analysis of the enabling technologies for next generation $5 \mathrm{G}$ wireless networks [9]. Many benefits of $5 \mathrm{G}$ may not be foreseen unless information and communications technology take into account the above challenges. Therefore, there are many important technical, regulatory and economical issues that should be considered in designing $5 \mathrm{G}$ wireless communication systems [7]. The standardization of $5 \mathrm{G}$ networks is expected to be around 2018 and start to deploy around 2020 [5]. Many new radio concepts such as massive multiple input multiple output (MIMO), ultradense networks, moving networks, M2M, and massive machine communications are considered to be the key components of $5 \mathrm{G}$ wireless networks. Heterogeneous network (HetNet) solutions are envisioned in $5 \mathrm{G}$ network, to order to achieve 1000 times the system capacity, 10 times the spectral efficiency, energy efficiency and peak data rate of $10 \mathrm{~Gb} / \mathrm{s}$ for low mobility and peak data rate of $1 \mathrm{~Gb} / \mathrm{s}$ for high mobility, and 25 times the average cell throughput needed $[9,10]$. In this HetNet solution, a mix of multiple wireless technologies, frequencies, cell sizes and hybrid network architectures are used to optimally respond to rapid changes in mobile user as well as multi-vendor, multiservice provider demand. In [11], co-existence of legacy radio 


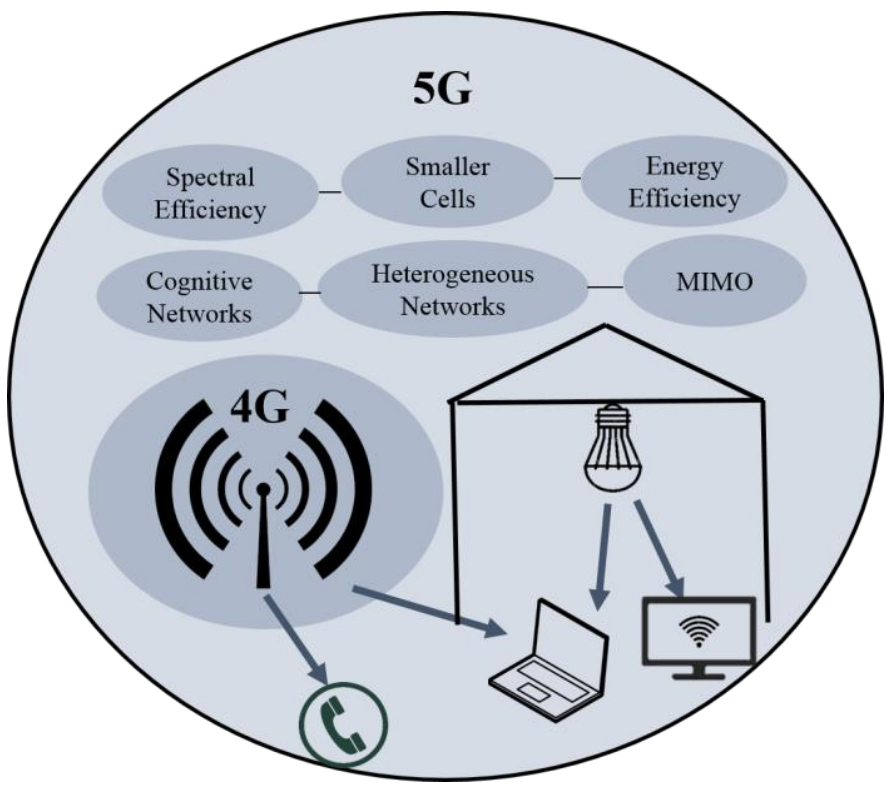

Figure 1. Components expected for the next generation of wireless communication. VLC has significant potential to be used as a supplementary technology to aid in achieving 5G telecommunications.

access technologies (RAT) and possible integration of new wireless access technologies such as visible light communication (VLC) and $\mathrm{mm}$ wave $(60 \mathrm{GHz})$ technology have been proposed to meet the demand of spectrum scarcity for future wireless networks, as shown in Figure 1. The effect of the unlicensed and unregulated spectrum of VLC and mm wave on the mobile business is not yet known, but approximately every $10 \mathrm{MHz}$ of additional licensed spectrum leads to a 263 million increase in wireless applications and content sales [12]. Additional benefits of such integration are reliable connectivity, higher throughput, better coverage and improved energy efficiency.

Lighting is a major source of electric energy consumption, accounting for around 19\% of indoor electricity usage [13]. Hence, development of more efficient lighting sources is important for energy conservation. As a result, a significant global movement toward replacing incandescent and fluorescent lights with solid state white LEDs (WLED) is underway. In addition to lighting capabilities, WLEDs could also be used to provide wireless communications by modulating the intensity of the light source faster than the human eye can perceive $[14,15]$. It is possible to turn any LED light fixture into a light transceiver, to serve as sources for even very high data rate applications. The dual use of LEDs for illumination and communication purposes is a sustainable and energy-efficient approach and has the potential to revolutionize how we use light. VLC can be possibly used in a wide range of applications including wireless local area networks, wireless personal area networks and vehicular networks among others.

\section{VISIBLE LIGHT COMMUNICATIONS}

Optical wireless $(\mathrm{OW})$ communication that enables wireless connectivity using infrared, visible or ultraviolet bands, and VLC is simply a subset of the OW field [17]. A basic VLC link is composed of a transmitting device modulating the illumination intensity (LED) with air as the propagation channel and a positive intrinsic-negative (PIN) or the more sensitive avalanche photodiode (APD) as the receiver. Such a link is often referred to as Li-Fi (Light Fidelity) [18]. VLC can provide access to several hundred terahertz (THz) of unlicensed spectrum. One of the key merits of optical wireless systems is the relatively low transceiver complexity and low energy-perbit required for data transmission in comparison to RF systems. Optical wireless systems operate using low power baseband hardware. Since visible light spectrum extends from $380 \mathrm{~nm}$ to $780 \mathrm{~nm}$ in wavelength, the VLC spectrum has immunity to electromagnetic interference and noninterference with RF systems. There are already several standards associated with visible light communications [19-21]. In 2007, the Japan Electronics and Information Technology Industries Association (JEITA) established standards and in 2008 the Visible Light Communications Consortium (VLCC) introduced specification standards [21]. In 2011, VLC was standardized and published as the IEEE 802.15.7 standard [21]. The standard includes specifications on networks capable of delivering $5 \mathrm{G}$ compatible data rates which is sufficient to support audio and video multimedia services and also considers the mobility of the visible link [21]. The IEEE 802.17.7 standard supports a device discovery mechanism through which short range co-operation can also be possible within homogeneous networks with three different classes of devices: infrastructure, mobile, and vehicle.

The potential advantages achieved by using VLC are numerous; however, this does not imply that VLC should be a replacement for RF technologies such as cellular, WLAN, Zigbee, UWB, and Bluetooth. Rather, applications including OW systems are very limited when considering area coverage and user mobility where RF technologies prove invaluable. Moreover, communication with VLC is challenging due to occlusion which can occur if severe misalignments and presence of physical obstruction between sources and detectors are present in indoor as well as outdoor environments. Visible light waves predominantly obey line of sight (LOS) propagation. Hence, VLC is more vulnerable than RF in terms of reliable connectivity especially when the mobile users move. The key merit OW exhibits are several appealing attributes when compared to RF. OW links are inherently broadband and optical frequencies in the infrared and visible spectrum are neither regulated nor licensed. Optical components are also cheaper and consume less electrical power than high-speed RF components. Finally, OWC has much less potential for interference with RF-sensitive electronic systems.

These characteristics generate substantial potential for VLC to be used as a complimentary technology with RF systems in the quest for $5 \mathrm{G}$ telecommunication. Given these advantages, 
we expect many more groups within the RF wireless community to venture into hybrid RF/OW experimental efforts in the near future (as we have recently done). In this context, we offer this report as a unique blend of review, initial experimental testbed observations, and visionary moving forward. Our hope is to support and aid RF groups as they transition their research interests to include RF/VLC hybrid networks. The main purpose of such integration between different wireless technologies is to exploit the benefits of each technology in such a way that they complement each other. The possible benefits may be identified as reliable connectivity, higher throughput, better coverage and improved energy efficiency [8].

\section{RELATED WORK}

The RF and VLC communities have frequently published stand-alone papers, but very few hybrid systems have been introduced. For the reasons stated above, we believe VLC is an ideal complimentary technology for the existing (and future) RF infrastructure, but is impractical in stand-alone VLC networks. A brief review of the recent developments within the VLC field will be given, followed by a discussion of the few reported hybrid RF/VLC systems.

Numerous findings are reported in the VLC literature intending to improve data rate capacity, especially for LOS link configurations [29-39]. Most of these works are related to hardware demonstrations where different modulation schemes, blue filtering, pre and post equalizer are used to improve the data rates. For example in [25], $125 \mathrm{Mbps}$ over $5 \mathrm{~m}$ distance in in-door environment by using OOK is reported. In the experimental setup, a lamp with six chips provides luminous flux of about $400 \mathrm{~lm}$, and a blue filter is applied to suppress the phosphorescent component of the white light. A large area silicon PIN diode with effective area $100 \mathrm{~mm}^{2}$ and polymer lens with $70^{\circ}$ FOV are used to receive the signal. The BER performance is measured by keeping luminance level at about 800 lux at the receiver. It is observed that the data rate is 80 Mbps at the corresponding BER 10-6. Another group reported a $513 \mathrm{Mbps}$ point-to-point visible light communication link using DMT modulation of white LED in [27]. Further improvement in data rate is continued by this group and achieved $803 \mathrm{Mbps}$ and reported in [34]. In this demonstration WDM VLC link with DMT modulation is used for RGB LEDs. In the receiver large area commercially available APD of $3 \mathrm{~mm}$ diameter combined with a glass lens of $8 \mathrm{~mm}$ focal length is used for detection. The gross transmission rates were about 293.7 Mbps, 223.4 Mbps and 286 Mbps for the red, green and blue channels respectively, which lead to aggregate data rate of about 803 Mbps. The milestone for Gbps data rate in optical wireless communication is reported in [29-31]. In [29], the first Gbps VLC link based on RGB LEDs was demonstrated. In this work, QAM on DMT is used in WDM link. DMT signals consisted of 128 subcarriers within the baseband bandwidth of 100MHz. Large area silicon APD with $3 \mathrm{~mm}$ diameter combined with a glass lens of $20 \mathrm{~mm}$ diameter and $20 \mathrm{~mm}$ focal length is used for detection. The detector is followed by a lowimpedance amplifier to amplify the signal level up to the operation range. The transmission performance measurements were performed with and without the presence of crosstalk. In case of without crosstalk, the gross transmission rate were about $444 \mathrm{Mbps}, 518 \mathrm{Mbps}$ and $589 \mathrm{Mbps}$ for the red, green and blue channel respectively which lead to aggregate data rate of about $1.55 \mathrm{Gbps}$. On the other hand, in case of with crosstalk the gross transmission rates were about $376 \mathrm{Mbps}, 439 \mathrm{Mbps}$ and 430 Mbps for the red, green and blue channels respectively, which lead to aggregate data rate of about $1.25 \mathrm{Gbps}$. $1 \mathrm{Gbps}$ for single

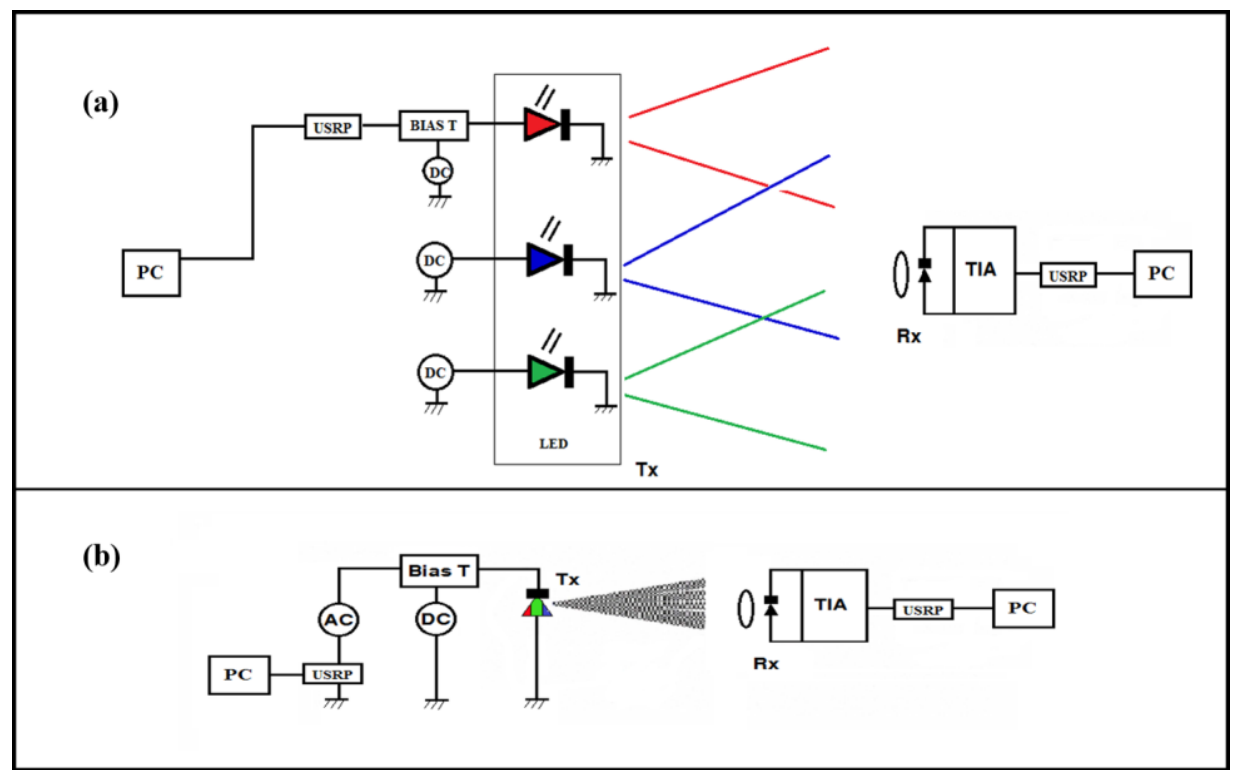

Figure 2. Schematic of VLC downlink for the (a) RGB LED, and (b) blue-phosphor LED. Each color in the RGB was successfully modulated, however, for initial testing the red LED was typically modulated due to the high responsivity of the PIN photodiode at that portion of the spectrum. 
channel and 2.1 Gbps WDM transmission at usual illumination levels are also reported in [31].

A key area in future OW/RF hybrid systems will be effective vertical handover (VHO) from $\mathrm{OW}$ to $\mathrm{RF}$ when the communication path of the OW transmission is obstructed, and vice versa when a clear link is established. This has been the main focus in recent papers that have proposed schemes for $\mathrm{VOH}$ to achieve optimal throughput and QoS [proposal $29,44,47]$.

\section{TestBed Design}

An ideal RF/VLC testbed should have a straightforward assembly, be versatile enough to allow for rapid exchange of emitter and receiver components, and facilitate many different modulation schemes. Unfortunately, most stand-alone VLC links, thus far reported, require extensive software and sometimes hardware development prior to successful operation. Consequently, these systems take substantial time to deploy and are typically limited in scope, allowing for only a limited number of experimental configurations. An exception to this trend are two reported systems found using software defined VLC links. Such a network is favorable for an adaptable experimental testbed, and, for this reason, was selected for use in the current study. Our software defined RF/OW hybrid network uses the open-source GNU Radio development toolkit, previously developed for signal processing characterization in software defined radio (SDR) systems. GNU Radio contains a database of different code-blocks allowing for easy interaction with external hardware components and quick adaption between modulation schemes. Our testbed uses GNU radio to interact with two N210 Universal Software Radio Peripherals (USRP2) from Ettus Research. The USRP2 hardware platform is composed of one of several interchangeable daughterboards, simple communication processers, and digital-to-analog converters (DAC) on a basic motherboard. This allows the units to act as an interface between the computer and the emitter/receiver. A BasicTX transmitter and BasicRX receiver daughterboards also from Ettus Research were used (1-250 $\mathrm{MHz}$ ). Figure 2 shows a schematic of our VLC downlink. A digital signal sent from the transmitting computer is digitally up converted in the first USRP and then passed through an inbuilt digital-to-analog converter. The resultant analog signal is then translated by the daughterboard to the transmitting frequency before propagating along the channel, which in a VLC link is air. The optical receiver filters the incoming radiation rejecting the unactuated broadband light and passing the narrowband transmitter radiation. A transimpedance amplifier is then used to create an electric analog signal, which is then converted by the USRP to a digital signal. As shown in Figure 4, the two main approaches to producing white light from LEDs are characterized in this study. A commercial RGB LED (LED ENGIN, 03MC00, $40 \mathrm{~W}, 81 \mathrm{~mm}$ footprint) and a blue-phosphor LED (LUXEON, LMZ7-xW30) were used. For the receiver, a PDA36A Thorlabs photodetector with the Thorlab-ALC2520A condensing lens was used (unless specified otherwise, $\varnothing 25$ $\mathrm{mm}, \mathrm{f}=20 \mathrm{~mm}$ ). This particular photodiode had a $340 \mathrm{~nm}-$ $1100 \mathrm{~nm}$ responsivity, a peak responsivity of $0.65 \mathrm{~A} / \mathrm{W}$ at 970 $\mathrm{nm}$, and a $13 \mathrm{~mm}^{2}$ active area. To complete the hybrid network, a basic RF uplink was combined with the VLC downlink. While we anticipate having a hybrid RF/OW downlink system in future designs, for the initial testbed characterization the different technologies were isolated to one specific function (i.e. uplink/downlink). Two basic VERT 400 (Ettus Research) USRP antennas were used for the RF component.

\section{RESUlts AND Dicussion}

The many different modulation schemes developed for RF signal processing have been leveraged in VLC links and can largely be categorized as either a pulsed modulation of the baseband (e.g. on-off-keying (OOK) and pulsed-positionmodulation (PPM)), or intensity modulation of the passband (e.g. amplitude-shift-keying (ASK), quadrature-amplitudemodulation (QAM), and Gaussian minimum shift keying (GMSK)). As in RF telecommunication networks, the selected modulation scheme plays an important role in determining the system throughput capacity. While several modulation schemes were tested (e.g., OOK, PPM), Gaussian minimum shift keying (GMSK) performed the best and is used for all the data reported in this work. The maximum data rates at different distances for both LED types can be seen in Figure 3. As expected, increased link distance decreased the observed bitrate. This can be attributed to less light entering the receiver at larger distances. When distance is not an issue, the maximum data rates realized were $6.25 \mathrm{Mb} /$ and $12.5 \mathrm{Mb} / \mathrm{s}$ for the blue-phosphor and RGB LEDs, respectively. It is important to note that only the red LED in the RGB scheme was modulated for the data recorded in Figure 3. All three

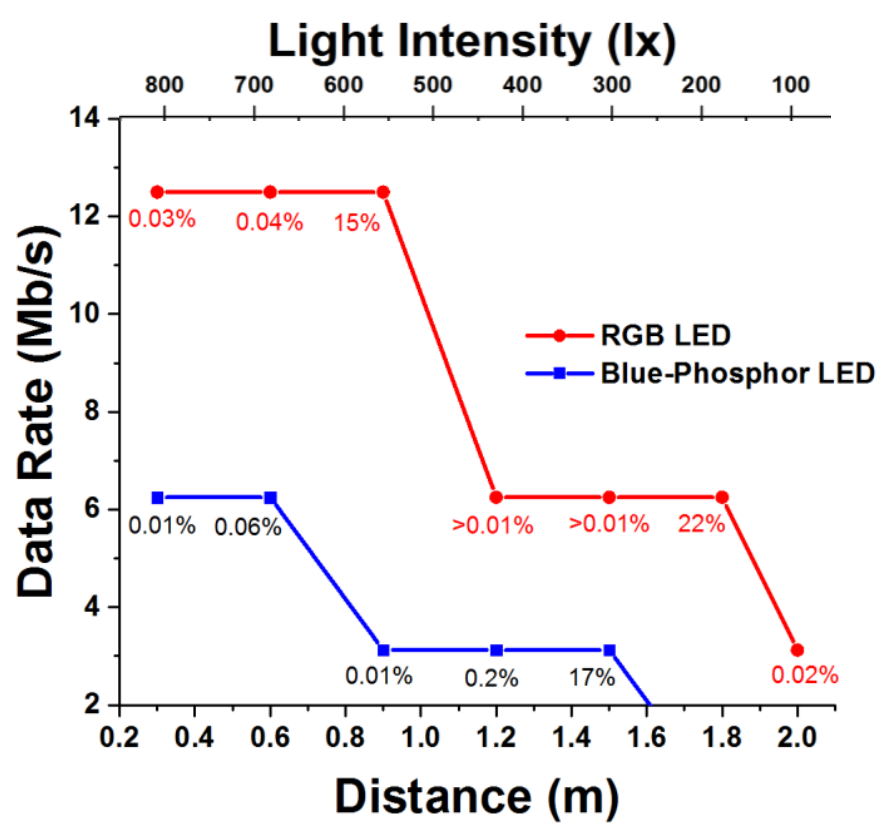

Figure 3. Maximum data rate performance of each LED type at different distances (and corresponding light intensities). The percentage next to each data point represents the packet error rate associated with that experiment. 

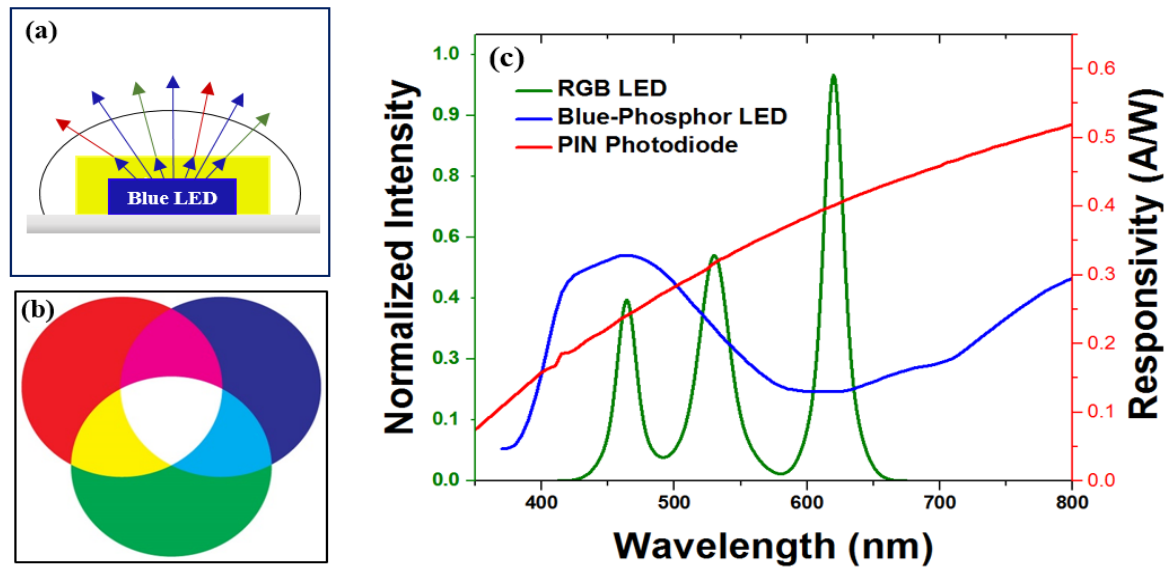

Figure 4. A (a) diagram of the composition of a blue-phosphor LED, (b) red-green-blue color blending used in RGB LEDs to produce white light, and (c) emission spectrum of the RGB LED (green), and blue Phosphor LEDs (blue), plotted with the responsivity spectrum of the PIN photodiode (red).

colors comprising the RGB LED were successfully modulated. A slight decreased in performance was observed when only using the blue color, for reasons later discussed. Theoretically, all three colors could be modulated simultaneously for triple the bitrate up to $37.5 \mathrm{Mb} / \mathrm{s}$. At data rates above $12.5 \mathrm{Mb} / \mathrm{s}$ the computers connected to the USRPs could not run at sufficient speeds to provide valid data. A cable connected between USRPs as a control (in place of the VLC link) was also constrained to $12.5 \mathrm{Mb} / \mathrm{s}$. To the best of our knowledge, 4 $\mathrm{Mb} / \mathrm{s}$ is the highest previously reported data-rate in a SDVLC link [35]. This increase in performance can be attributed to use of GMSK as the modulation mechanism and use of a RGB LED; the few other reported SDVLC links in the literature employ blue-phosphor LEDs for simultaneous data transfer and illumination.

Due to slow response rates of the phosphors required to produce white light in blue-phosphor LEDs, their modulation bandwidths are more constricted than RGB LEDs. To combat this issue, researchers $[3.4 \mathrm{~Gb} / \mathrm{s}$ paper] have installed blue filters over the receiver preventing any phosphorescent generated light from interfering with the blue LED signal. While this has been proven to increase data rates, higher illumination levels are required, reducing power efficiencies. Our attempt to use a 390-480 nm blue filter (Thorlabs- FD1B) with the blue-phosphor LED for higher data rates proved unsuccessful due to the low responsivity performance of our photodiode in the blue region, as seen in Figure 4. The blue LED emission maximum at $\sim 450 \mathrm{~nm}$ corresponds with a 0.3 $\mathrm{A} / \mathrm{W}$ responsivity in the photodiode, whereas at longer wavelengths the responsivity is up to $0.9 \mathrm{~A} / \mathrm{W}$. This decreased sensitivity combined with the decreased power efficiency requires dangerously high illumination values $(+1100 \mathrm{~lx})$, so blue filter was not included in most experiments. For the same reasons, data transfer via modulating the blue color in the RGB LED is less effective than green or red. A more sensitive PIN photodiode, or even better, the use of an avalanche photodiode would eliminate this obstacle and are anticipated to be used in future designs. These results are still significantly lower than VLC links with custom software and hardware that have achieved several gigabit per second throughputs; however, more spectrally efficient modulation techniques like orthogonal frequency division multiplexing (OFDM) or discrete multi-tone (DMT) schemes have far higher data rates. Aside from implementing new modulation and multiplexing schemes, all three RGB LEDs will be modulated simultaneously to obtain combined data rate observations. Our future work on this testbed will be to develop a more practical hybrid software defined OW/RF hybrid that includes a vertical handover scheme from OW to RF to allow for increased mobility.

As we progress towards the heavy inflation of connected user devises with the ever expanding IoT, efficient access points will be needed. In recent years, the most impressive capacity gains have been achieved by bringing access points closer to UDs to reduce cell size. As the density of connected devices per a given area increases, more and closer access points are needed. Hyperdense or directed access points will improve the local area spectral efficiency $\left(\mathrm{b} / \mathrm{s} / \mathrm{m}^{2}\right)$. If a VLC link is a component in this heterogeneous network, then

\section{VISIONARY}

Even though the standardization and the commercialization of the $4 \mathrm{G}$ networks was recent the research community is already working on enabling the next generation wireless technologies (5G). This new technology will aim for efficiency in spectrum use, handling of massive amount of data mainly in short distances, less carbon footprint, personalized APs, and high data rates. To meet these challenges, the current $4 \mathrm{G}$ networks need to expand into smaller cells, directional wireless, and heterogeneous networks comprised of many different sized cells. Cognitive radio, software defined radio networks (SDN), 


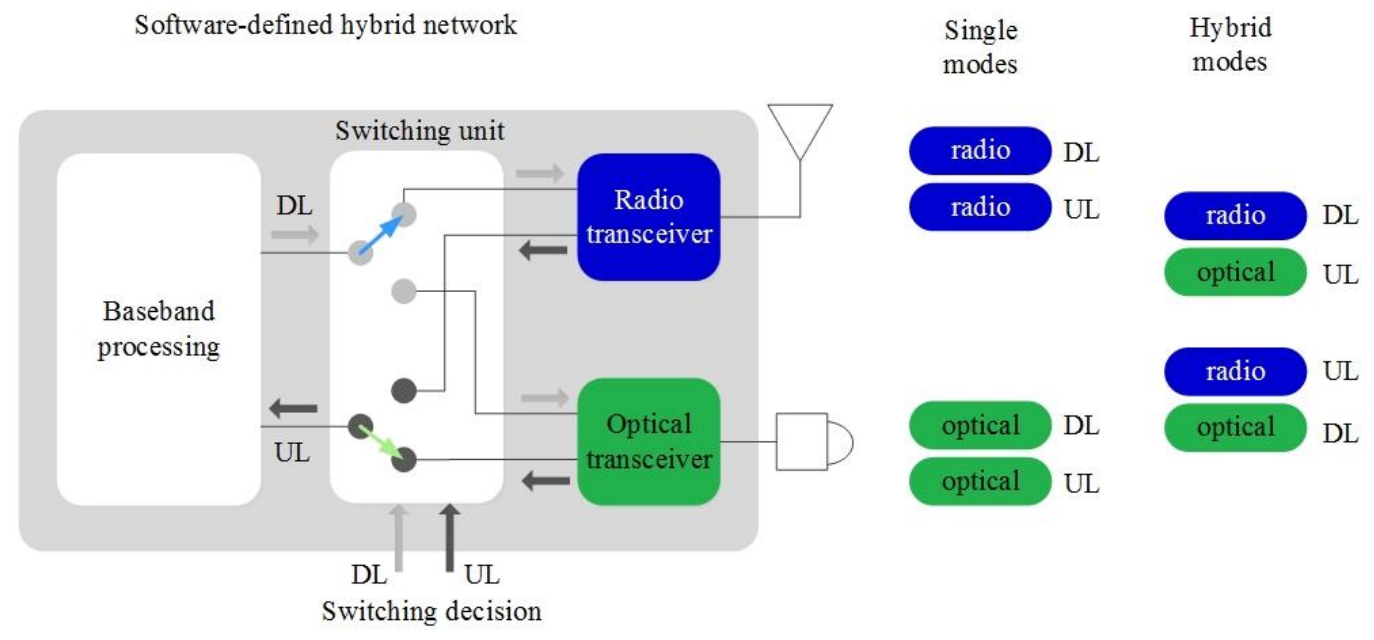

Figure 5. Software defined hybrid network handover scheme

duplex devices that can transmit and receive in the same or multiple frequency simultaneously, and massive multipleinput multiple-output (MIMO) systems are also considered key research areas in the push towards $5 \mathrm{G}$.

A HetNet system mainly based on VLC/ RF hybrid system is capable of meeting all the $5 \mathrm{G}$ requirements, providing immense amounts of data at high rates in small distances, and providing personalized links while serving for both illumination and communication purposes, thus increasing the efficiency. VLC links offer high data rates in unlicensed bandwidth but are severely limited by user mobility. These links when operating as full modes are not reliable; therefore, hybrid system where VLC is used in conjunction with RF are proposed to offer both high data rates and reliability. The RF link might be used for backup, maintenance, and control purposes while VLS is used to provide the bulk of data. All these functionalities require low line rate, therefore the unlicensed bands at $2.4 \mathrm{GHz}, 5 \mathrm{GHz}$ and $6 \mathrm{GHz}$ can be used. Our proposed link, displayed in Figure 5, is a Hybrid VLC/RF link that allows for switching and data splitting between the two links. Depending on the quality of each link the system allows for four possible combinations. Normally the link would serve as a full VLC mode, but in case this link fails to meet a threshold data rate the system will move to full mode RF. When the VLC link can reach a data rate above the threshold the system will be switch back. In another instance when wither uplink or downlink VLC fails, this link will be switched to RF to provide data transmission. The moment the replaced link becomes available it is switched back again. In all the above scenarios, RF is used as a backup link (due to the reliable nature of RF connections). On the same note, RF can be used as a parallel link with VLC. In this instance the system can operate as full mode VLC and full mode RF which is in congruence with HetNEt systems for the next generation wireless technologies $(5 \mathrm{G})$.

\section{CONCLUSION}

To the best of our knowledge, $12.5 \mathrm{Mb} / \mathrm{s}$ represents the highest reported data rate for a software defined VLC downlink and is the first employing a RF uplink. More importantly, the experimental testbed described within represents an adaptable platform to begin hybrid RF/OW work. We hope this work will encourage and guide traditionally RF groups to venture into hybrid systems, which have expanding potential to provide many of the solutions needed to realize $5 \mathrm{G}$ telecommunication. As more attention is drawn to hybrid systems, we also anticipate more hardware design and optimization. Current LEDs and photodetectors were not designed to serve as VLC system components. While VLC is not recommended for stand-alone use, it is well suited to compliment RF networks as a new high-speed layer in a heterogeneous network.

\section{ACKNOWLEDGMENTS}

The authors would like to thank the CWC and the University of Oulu for their support and funding. Thanks to Drexel University for personal funding. We are tremendously grateful to Dr. Adam Fontecchio in the College of Engineering for connecting us to the University of Oulu (and support through the Paul Peck Scholars Program), for Dr. Suzanne Rocheleau, and Jaya Mohan for their continued personal funding/support through the iSTAR program, and to Holly Burnside and Alistar Erickson-Ludwig at Drexel for their efforts in making our stay in Finland possible. We would also like to thank Sara Braun for her efforts in this work.

\section{REFERENCES}

[1] Hanzo L, Haas H, Imre S, O'brien D, Rupp M \& Gyongyosi L (2012) Wireless myths, realities, and futures: From $3 \mathrm{~g} / 4 \mathrm{~g}$ to optical and quantum wireless. Proceedings of the IEEE 100(Special Centennial Issue): 18531888.

[2] Chowdhury H, Pouttu A \& Katz M (2014) Performance analysis of visible light communications-based hotspots in indoor and outdoor environments. Wireless personal communications, Springer (to appear) . 
[3] Kavehrad M (2010) Sustainable energy-efficient wireless applications using light. Communications Magazine, IEEE 48(12): 66-73.

[4] Chowdhury H, Ashraf I \& Katz M (2013) Energy-efficient connectivity in hybrid radio-optical wireless systems. In: Wireless Communication Systems (ISWCS 2013), Proceedings of the Tenth International Symposium on, pp. 1-5.

[5] Olsson M, Cavdar C, Frenger P, Tombaz S, Sabella D \& Jantti R (2013) 5green: Towards green $5 \mathrm{~g}$ mobile networks. In: Wireless and Mobile Computing, Networking and Communications (WiMob), 2013 IEEE 9th International Conference on, pp. 212-216.

[6] Radwan A \& Rodriguez J (2012) Energy saving in multi-standard mobile terminals through short-range cooperation. EURASIP J. Wireless Comm. and Networking 2012: 159.

[7] Chowdhury H, Bagheri H, Ashraf I, Tamoor-ul Hassan S \& KatzM(2013) Techno-economic analysis of visible light communications. In: Wireless Communication Systems (ISWCS 2013), Proceedings of the Tenth International Symposium on, pp. 1-5.

[8] Chowdhury H \& Katz M (2014) Cooperative data download on the move in indoor hybrid (radio-optical) wlan-vlc hotspot coverage. Transactions on Emerging Telecommunications Tecnologies, John Wiley Sons 25(7): 666-677.

[9] Osseiran A, Boccardi F, Braun V, Kusume K, Marsch P, Maternia M, Queseth O, Schellmann M, Schotten H, Taoka H, Tullberg H, Uusitalo M, Timus B \& Fallgren M (2014) Scenarios for 5g mobile and wireless communications: the vision of the metis project. Communications Magazine, IEEE 52(5): 26-35.

[10] Mogensen P, Pajukoski K, Tiirola E, Lahetkangas E, Vihriala J, Vesterinen S, Laitila M, Berardinelli G, Da Costa G, Garcia L, Tavares F \& Cattoni A (2013) 5g small cell optimized radio design. In: Globecom Workshops (GC Wkshps), 2013 IEEE, pp. 111-116.

[11] Wang CX, Haider F, Gao X, You XH, Yang Y, Yuan D, Aggoune H, Haas H, Fletcher S \& Hepsaydir E (2014) Cellular architecture and key technologies for $5 \mathrm{~g}$ wireless communication networks. Communications Magazine, IEEE 52(2): 122-130.

[12] URI: http://apps.fcc.gov/ecfs/document/view?id=7022123551 .

[13] Wu Z \& Little T (2010) Network solutions for the los problem of new indoor free space optical system. In: Communication Systems Networks and Digital Signal Processing (CSNDSP), 2010 7th International Symposium on, pp. $582-587$.

[14] Minh HL, Ghassemlooy Z, O'Brien D \& Faulkner G (2010) Indoor gigabit optical wireless communications: Challenges and possibilities. In: Transparent Optical Networks (ICTON), 2010 12th International Conference on, pp. $1-6$.

[15] Langer KD, Grubor J, Bouchet O, El Tabach M,Walewski J, Randel S, Franke M, Nerreter S, O’Brien D, Faulkner G, Neokosmidis I, Ntogari G \& Wolf M (2008) Optical wireless communications for broadband access in home area networks. In: Transparent Optical Networks, 1272008. ICTON 2008. 10th Anniversary International Conference on, volume 4, pp. 149-154.

[16] URI: http://irdajp.info/specifications.html .

[17] Elgala H, Mesleh R \& Haas H (2011) Indoor optical wireless communication: potential and state-of-the-art. Communications Magazine, IEEE 49(9): 56-62.

[18] O’Brien D, Faulkner G, Jim K, Zyambo E, Edwards D, Whitehead M, Stavrinou P, Parry G, Bellon J, Sibley M, Lalithambika V, Joyner V, Samsudin R, Holburn D \& Mears R (2003) High-speed integrated transceivers for optical wireless. Communications Magazine, IEEE 41(3): $58-62$.
[19] CP-1221 (2007) Japan electronics industry technology association. In: Japanese Visible Light Communications System.

[20] CP-1222 (2007) Japan electronics industry technology association. In: Japanese Visible Light ID System.

[21] IEEE1 (2011) Ieee standard for local and metropolitan area networkspart 15.7: Short-range wireless optical communication using visible light. IEEE Std 802.15.7-2011 pp. 1-309.

[22] Minh HL, O’Brien D, Faulkner G, Zeng L, Lee K, Jung D \& Oh Y (2008) High-speed visible light communications using multiple-resonant equalization. Photonics Technology Letters, IEEE 20(14): 1243 -1245.

[23] Bouchet O, Porcon P,Wolf M, Grobe L,Walewski J, Nerreter S, Langer K, Ferna? andndez L, Vucic J, Kamalakis T, Ntogari G \& Gueutier E (2010) Visible-light communication system enabling $73 \mathrm{mb} / \mathrm{s}$ data streaming. In: GLOBECOM Workshops (GC Wkshps), 2010 IEEE, pp. $1042-1046$.

[24] Minh HL, O’Brien D, Faulkner G, Zeng L, Lee K, Jung D \& Oh Y (2008) $80 \mathrm{mbit} / \mathrm{s}$ visible light communications using pre-equalized white led. In: Optical Communication, 2008. ECOC 2008. 34th European Conference on, pp. $1-2$.

[25] Vucic J, Kottke C, Nerreter S, Habel K, Buttner A, Langer KD \& Walewski J (2009) $125 \mathrm{mbit} / \mathrm{s}$ over $5 \mathrm{~m}$ wireless distance by use of ookmodulated phosphorescent white leds. In: Optical Communication, 2009. ECOC '09. 35th European Conference on, pp. $1-2$.

[26] Vucic J, Kottke C, Nerreter S, Habel K, Buttner A, Langer KD \& Walewski J (2010) $230 \mathrm{mbit} / \mathrm{s}$ via a wireless visible-light link based on ook modulation of phosphorescent white leds. In: Optical Fiber Communication (OFC), collocated National Fiber Optic Engineers Conference, 2010 Conference on (OFC/NFOEC), pp. 1 -3.

[27] Vuc J, Kottke C, Nerreter S, Langer K \& Walewski J (2010) 513 mbit/s visible light communications link based on dmt-modulation of a white led. Lightwave Technology, Journal of 28(24): $3512-3518$.

[28] Vucic J, Kottke C, Habel K \& Langer KD (2011) 803 mbit/s visible light wdm link based on dmt modulation of a single rgb led luminary. In: Optical Fiber Communication Conference and Exposition (OFC/NFOEC), 2011 and the National Fiber Optic Engineers Conference, pp. $1-3$.

[29] Kottke C, Hilt J, Habel K, Vucic J \& Langer KD (2012) 1.25 gbit/s visible light wdm link based on dmt modulation of a single rgb led luminary. In: Optical Communications (ECOC), 2012 38th European Conference and Exhibition on, pp. 1-3.

[30] Cossu G, Corsini R \& Ciaramella E (2014) High-speed bi-directional optical wireless system in non-directed line-of-sight configuration. Lightwave Technology, Journal of 32(10): 2035-2040.

[31] Minh HL, O’Brien D, Faulkner G, Bouchet O, Wolf M, Grobe L \& Li J (2010) A $1.25-\mathrm{gb} / \mathrm{s}$ indoor cellular optical wireless communications demonstrator. Technology Letters, IEEE 22(21): 1598 -1600.

[32] Minh HL, O’Brien D \& Faulkner G (2010) A gigabit/s indoor optical wireless system for home access networks. In: Communication Systems Networks and Digital Signal Processing (CSNDSP), 2010 7th International Symposium on, pp. 532-536.

[33] Minh HL, O’Brien D, Faulkner G, Bouchet O, Wolf M, Grobe L \& Li J (2010) A 1.25- gb/s indoor cellular optical wireless communications demonstrator. Photonics Technology Letters, IEEE 22(21): 1598 -1600.

[34] M. Rahaim, T. Borogovac, T.D.C. Little, Ali Mirvakili, V. Joyner, "Demonstration of a Software Defined Visible Light Communication System," in MCL Technical Report No. 09-15-2011, Sept. 2011.

[35] Calvin Ball, Kevin Tien, "Desgin and Development of a Visible Light Communications Link", Department of Electrical Engineering, The Cooper Union for the Advancement of Science and Art . 